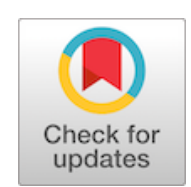

\title{
Lesión pancreática aislada tras traumatismo abdominal cerrado: a propósito de un caso
}

\author{
Isolated pancreatic injury after blunt abdominal trauma: a case report
}

\author{
Teresa Carrascosa-Mirón¹, Alberto Carabias-Hernández¹, Javier Rodríguez-Padilla², \\ Alba Manuel-Vázquez³ , José María Jover-Navalón¹ \\ Servicio de Cirugía General y del Aparato Digestivo. Hospital Universitario de Getafe, Madrid, España. \\ 2 Servicio de Angiología y Cirugía Vascular. Hospital Universitario Severo-Ochoa, Madrid, España. \\ Servicio de Cirugía General y del Aparato Digestivo, Hospital Universitario de Guadalajara, Guadalajara, España.
}

\section{Resumen}

Introducción. La incidencia de lesiones traumáticas del páncreas es baja debido a su localización retroperitoneal, y las lesiones aisladas son excepcionales debido a la proximidad del páncreas a otros órganos y estructuras vasculares.

Caso clínico. Presentamos el caso de un varón de 21 años con lesión aislada del páncreas tras traumatismo abdominal cerrado, que precisó manejo quirúrgico.

Discusión. Las lesiones pancreáticas aisladas secundarias a traumatismo son raras, pero debemos tenerlas en cuenta en un paciente politraumatizado. Su diagnóstico precoz y manejo adecuado son cruciales para evitar el desarrollo de complicaciones.

Palabras clave: páncreas; heridas y traumatismos; diagnóstico; clasificación; procedimientos quirúrgicos; colangiopancreatografia retrógrada endoscópica.

\footnotetext{
Abstract

Introduction. The incidence of traumatic injuries to the pancreas is low due to its retroperitoneal location, and isolated injuries are rare due to the proximity of the pancreas to other vascular organs and structures.

Clinical case. We present the case of a 21-year-old man with an isolated lesion of the pancreas after blunt abdominal trauma, which required surgical management.

Discussion. Isolated pancreatic injuries secondary to trauma are rare but must be taken into account in a multiple trauma patient. Its early diagnosis and proper management are crucial to avoid the development of complications.

Keywords: pancreas; wounds and injuries; diagnosis; classification; surgical procedures; endoscopic retrograde cholangiopancreatography.

Fecha de recibido: 08/04/2020 - Fecha de aceptación: 18/05/2020 - Fecha de publicación en línea: 07/04/2021

Correspondencia: Teresa Carrascosa-Mirón, Carretera Madrid-Toledo, Km 12,500, 28905. Getafe, Madrid.

Teléfono: 0034647563340. Correo electrónico: t.carrascosa@hotmail.com

Citar como: Carrascosa-Mirón T, Carabias-Hernández A, Rodríguez-Padilla J, Manuel-Vázquez A, Jover-Navalón JM. Lesión pancreática aislada tras traumatismo abdominal cerrado: a propósito de un caso. Rev Colomb Cir. 2021;36:545-8. https://doi.org/10.30944/20117582.564 Este es un artículo de acceso abierto bajo una Licencia Creative Commons - BY-NC-ND https://creativecommons.org/licenses/by-nc$\mathrm{nd} / 4.0 /$ deed.es
} 


\section{Introducción}

La localización retroperitoneal del páncreas ejerce un efecto protector ante un traumatismo, lo que hace que las lesiones traumáticas del páncreas sean poco frecuentes, pero esa misma localización puede dificultar o retrasar el diagnóstico ${ }^{1,2}$.

Además, debido a la proximidad del páncreas a otros órganos y estructuras vasculares, las lesiones traumáticas del pancreas aisladas son excepcionales, sobre todo, cuando la lesión es producida por mecanismo penetrante ${ }^{3}$.

\section{Caso clínico}

Presentamos el caso de un hombre de 21 años, sin antecedentes relevantes, que acude a Urgencias tras traumatismo contuso por agresión, con múltiples lesiones en cara, miembros superiores y abdomen. A su llegada a Urgencias presentaba tensión arterial 130/74 mmHg y frecuencia cardiaca 90 lpm, y en la exploración física, dolor a la palpación de mesogastrio, con defensa abdominal, sin signos de irritación peritoneal. En la analítica se observó aumento en lipasa (9982 U/L) y leucocitosis $(14.500 / \mathrm{mm} 3)$, con hemoglobina normal $(14,5 \mathrm{~g} / \mathrm{dL})$.

Ante la persistencia del dolor abdominal, y dada la estabilidad hemodinámica, se realizó tomografía computarizada (TC) de abdomen y pelvis (figura 1), donde se observa laceración en cuerpo del páncreas, sin poder descartar afectación del conducto de Wirsung, y laceración hepática grado I de la AAST (American Association for the Surgery of Trauma $)^{4}$, con abundante líquido libre peripancreático, en ambas gotieras parietocólicas $\mathrm{y}$ en pelvis.

Se inició tratamiento conservador con líquidos endovenosos, analgesia y estrecha vigilancia clínica. Se amplió el estudio con colangiografía por resonancia magnética ( $\mathrm{C}-\mathrm{RM}$ ) (figura 1) para valorar conducto pancreático, donde se observó sección del cuerpo del páncreas, con lesión traumática del conducto pancreático principal (lesión grado III de la AAST).

Ante el aumento progresivo de dolor abdominal, los hallazgos radiológicos y la imposibilidad de realizar colangiopancreatografía retrógrada endoscópica (CPRE) de forma precoz, se decide intervención quirúrgica. Mediante laparotomía media, se observó gran hematoma peripancreático, en epiplón menor y mesocolon transverso, hematoma intrapancreático a nivel del cuello, con laceración en su cara anterior, lateral a la vena mesentérica superior, sin identificar conducto de Wirsung, y hemoperitoneo en pelvis de aproximadamente $200 \mathrm{ml}$, sin otras lesiones intraabdominales. Se decidió realizar pancreatectomía córporo-caudal con preservación esplénica, siguiendo la línea de la laceración, y cierre distal del páncreas con Prolene ${ }^{\circledR}$ y refuerzo con Tachossil ${ }^{\circledR}$ (figura 2). El paciente no presentó complicaciones en el postoperatorio inmediato.

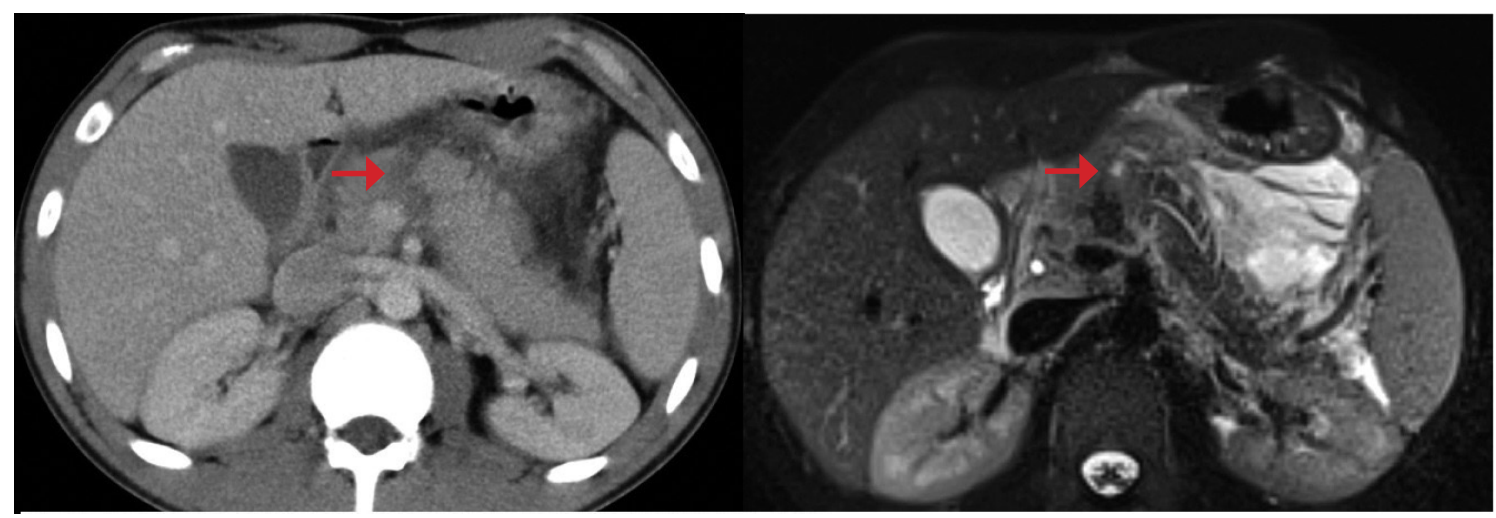

Figura 1. TC abdomino-pélvico y C-MR que muestran laceración en cuerpo del páncreas, grado III de la AAST. 
A los 7 días, presentó dolor abdominal y vómitos, junto con elevación de lipasa sanguínea (1697 U/L), por lo que se realiza nuevo TC abdomen, donde se aprecia colección peripancréatica de $11 \mathrm{~cm}$ en lecho de pancreatectomía, con impronta sobre cámara gástrica. Se realizó drenaje percutáneo (amilasa 2.462 U/L en el líquido), con buena evolución posterior. Fue dado de alta con drenaje a los 14 días (clasificación Clavien-Dindo IIIA).

Al mes tras el alta, se realizó resonancia magnética de abdomen, sin observar colecciones en el lecho quirúrgico, por lo que se decidió retirar el drenaje. La analítica mostró una normalización de las enzimas pancreáticas (lipasa $<70 \mathrm{U} / \mathrm{L}$, amilasa $<137 \mathrm{U} / \mathrm{L}$ ) y niveles elevados de glucosa en varias determinaciones ( $>200 \mathrm{mg} / \mathrm{dl}$ ), con lo que se diagnosticó diabetes mellitus y se inició tratamiento con insulina. Al año de seguimiento, el paciente se encuentra asintomático, con un excelente control glucémico (hemoglobina glicosilada $<6 \%$ ).

\section{Discusión}

Las lesiones traumáticas del páncreas aisladas son excepcionales y su diagnóstico es complejo, fundamentalmente debido a su localización.

Si el paciente está inestable hemodinámicamente, la única opción diagnóstica es la cirugía. Por el contrario, en pacientes hemodinámica-

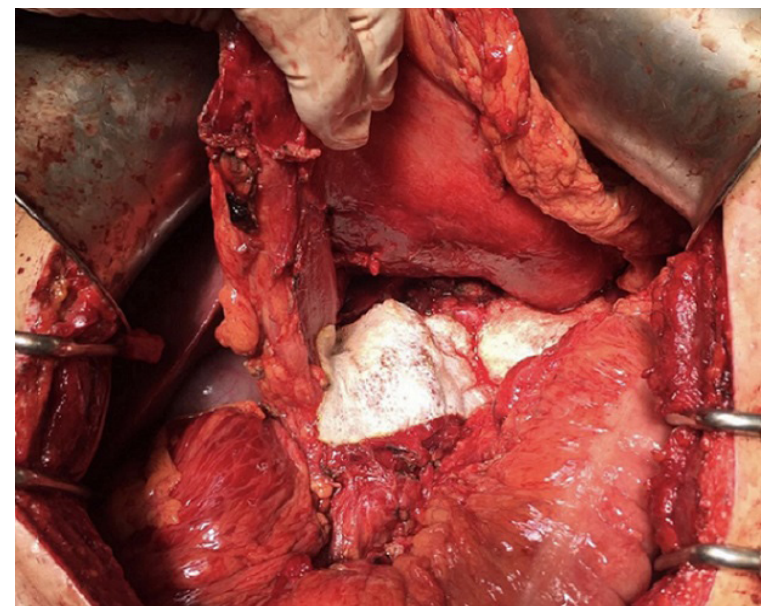

Figura 2. Campo quirúrgico, donde se observa la aplicación de Tachossil ${ }^{\circledR}$ sobre lecho pancreático. mente estables con lesión pancreática, las pruebas radiológicas deben dirigirse a la evaluación del conducto pancreático, ya que su lesión condiciona una mayor probabilidad de complicaciones, como son el pseudoquiste o la fístula pancreática. La lesión del conducto de Wirsung es el principal factor predictivo de morbimortalidad en traumatismos pancreáticos ${ }^{5,6}$.

A nivel radiológico, la TC permite excluir otras lesiones asociadas y podría aportar información acerca del conducto pancreático, pero su sensibilidad se sitúa en torno al $50 \%{ }^{7}$, un valor menor al obtenido por la C-RM y la CPRE.

En pacientes estables con lesión ductal o complicaciones tardías, el tratamiento endoscópico mediante la colocación de un stent transpapilar y/o esfinterotomía, podría evitar la cirugía ${ }^{8}$, por lo que la CPRE tiene un papel destacado en el diagnóstico y tratamiento en pacientes seleccionados ${ }^{4,9}$. El principal inconveniente es su falta de disponibilidad en determinados centros, como en nuestro caso, y la necesidad de personal con experiencia.

En cuanto al tratamiento, tras traumatismo contuso y lesión pancreática con conducto de Wirsung íntegro (grado I y II de las AAST), en paciente hemodinámicamente estable se recomienda, por lo general, tratamiento no quirúrgico ${ }^{7}$. Por el contrario, en pacientes inestables o con lesiones grado III, IV y V de la AAST, la cirugía es la mejor opción terapéutica.

La localización de la lesión y la identificación del conducto de Wirsung son los principales determinantes a la hora de seleccionar la técnica quirúrgica. Esta puede ir desde una pancreatorrafia, siempre y cuando se identifique el conducto íntegro, a una sutura primaria del Wirsung. El edema y el hematoma que se asocia a la lesión pancreática producen una alteración anatómica que dificulta e impide la identificación del Wirsung, lo que obliga a realizar técnicas estándar, como la pancreatectomía distal, como ocurrió en nuestro caso.

En conclusión, las lesiones pancreáticas traumáticas son raras, pero debemos tenerlas en cuenta ante un paciente politraumatizado. Su diagnóstico precoz y un buen manejo son cruciales para evitar el desarrollo de complicaciones y un desenlace fatal. 


\section{Cumplimiento de normas éticas}

Consentimiento del paciente: El paciente es informado de la peculariedad de su patología y de los hallazgos encontrados. Se le explica que su caso será enviado a una revista científica para su divulgación. Paciente acepta y nos autoriza para ello.

Conflicto de intereses: Los autores no hemos recibido financiación alguna ni declaramos conflicto de intereses alguno.

Financiación: Autofinanciado por los autores.

\section{Contribución de los autores:}

- Concepción y diseño del estudio: Teresa CarrascosaMirón.

- Adquisición de datos: Alberto Carabias-Hernández.

- Redacción del manuscrito: Teresa Carrascosa-Mirón, Alba Manuel-Vásquez y Javier Rodríguez-Padilla.

- Revisión crítica: Alberto Carabias-Hernández, José María Jover-Navalón.

\section{Referencias}

1. Wisner DH, Wold RL, Frey CF. Diagnosis and treatment of pancreatic injuries. An analysis of management principles. Arch Surg. 1990;125:1109-13. https://doi.org/10.1001/archsurg.1990.01410210035004

2. Sosa-Martin G, Morales-Portuondo K, Báez-Franco Z. Trauma pancreático. Rev. Cubana Cir. 2010;49(2). Fecha de consulta: 18 de febrero de 2020. Disponible en: http://scielo.sld.cu/pdf/cir/v49n2/cir12210.pdf
3. Iacono C, Zicari M, Conci S, Valdegamberi A, De Angelis $\mathrm{M}$, Pedrazzani $\mathrm{C}$, et al. Management of pancreatic trauma: A pancreatic surgeon's point of view. Pancreatology. 2016;16:302-8. https://doi.org/10.1016/j.pan.2015.12.004

4. Boffard D, ed. Manual of definitive surgical trauma care. $3^{\text {th }}$ edition. London: Hodder Arnold; 2011, pp. 118-9.

5. Lin BC, Chen RJ, Fang JF, Hsu YP, Kao YC, Kao JL. Management of blunt major pancreatic injury. J Trauma. 2004;56:774-8.

https://doi.org/10.1097/01.ta.0000087644.90727.df

6. Lochan R, Sen G, Barret AM, Scott J, Charnley RM. Management strategies in isolated pancreatic trauma. J Hepatobiliary Pancreatic Surg. 2009;16:189-96. https://doi.org/10.1007/s00534-009-0042-5

7. Petrone P, Moral-Alvarez S, González-Pérez M, Ceballos-Esparragón J, Marini CP. Orientación terapeútica del traumatismo pancreático: revisión de la literatura. Cir Esp. 2017:95:123-30. https://doi.org/10.1016/j.ciresp.2016.05.011

8. Thomson DA, Krige JEJ, Thomson SR, Bornman PC. The role of endoscopic retrograde pancreatography in pancreatic trauma: A critical appraisal of 48 patients treated at a tertiary institution. J Trauma Acute Care Surg. 2014;76:1362-6. https://doi.org/10.1097/TA.0000000000000227

9. Jeroukhimov I, Zoarets I, Wiser I, Shapira Z, Abramovich D, Nesterenko V, et al. Diagnostic use of endoscopic retrograde cholangiopancreatectography for pancreatic duct injury in trauma patients. Isr Med Assoc J. 2015;17:401-4. 